\title{
Strong Convergence of a New Three Step Iterative Scheme in Banach Spaces
}

\author{
Renu Chugh, Vivek Kumar*, Sanjay Kumar \\ Department of Mathematics, Maharshi Dayanand University, Rohtak, India \\ Email: *ratheevivek15@yahoo.com
}

Received August 11, 2012; revised October 12, 2012; accepted November 3, 2012

\begin{abstract}
In this paper, we suggest a new type of three step iterative scheme called the CR iterative scheme and study the strong convergence of this iterative scheme for a certain class of quasi-contractive operators in Banach spaces. We show that for the aforementioned class of operators, the CR iterative scheme is equivalent to and faster than Picard, Mann, Ishikawa, Agarwal et al., Noor and SP iterative schemes. Moreover, we also present various numerical examples using computer programming in $\mathrm{C}++$ for the $\mathrm{CR}$ iterative scheme to compare it with the other above mentioned iterative schemes. Our results show that as far as the rate of convergence is concerned 1) for increasing functions the CR iterative scheme is best, while for decreasing functions the SP iterative scheme is best; 2) CR iterative scheme is best for a certain class of quasi-contractive operators.
\end{abstract}

Keywords: Fixed Point; Various Iterative Schemes; Rate of Convergence

\section{Introduction}

There is a close relationship between the problem of solving a nonlinear equation and that of approximating fixed points of a corresponding contractive type operator. Consequently, there is theoretical and practical interest in approximating fixed points of various contractive type operators. Let $(X, d)$ be a complete metric space and $T: X \rightarrow X$ a self map for $X$. Suppose that $F(T)=\{p \in X, T p=p\}$ is the set of fixed points of $T$. There are several iterative processes in the literature for which the fixed points of operators have been approximated over the years by various authors.

In a complete metric space, the Picard iterative scheme $\left\{x_{n}\right\}_{n=0}^{\infty}$, defined by

$$
x_{n+1}=T x_{n}, n=0,1, \cdots
$$

has been employed to approximate the fixed points of mappings satisfying the inequality

$$
d(T x, T y) \leq \alpha d(x, y)
$$

for all $x, y \in X$ and $\alpha \in[0,1)$.

Condition (1.2) is called the Banach's contraction condition. Any operator satisfying (1.2) is called a strict contraction.

In 1953, W. R. Mann defined the Mann iterative scheme [1] as

$$
u_{n+1}=\left(1-\alpha_{n}\right) u_{n}+\alpha_{n} T u_{n},
$$

\footnotetext{
${ }^{*}$ Corresponding author.
}

where $\left\{\alpha_{n}\right\}$ is a sequence of positive numbers in $[0,1]$.

In 1974, S. Ishikawa defined the Ishikawa iterative scheme [2] as

$$
\begin{aligned}
& s_{n+1}=\left(1-\alpha_{n}\right) s_{n}+\alpha_{n} T t_{n} \\
& t_{n}=\left(1-\beta_{n}\right) s_{n}+\beta_{n} T s_{n},
\end{aligned}
$$

where $\left\{\alpha_{n}\right\}$ and $\left\{\beta_{n}\right\}$ are sequences of positive numbers in $[0,1]$.

In 2007, Agarwal et al. defined the Agarwal et al. iterative scheme [3] as

$$
\begin{gathered}
s_{n+1}=\left(1-\alpha_{n}\right) T s_{n}+\alpha_{n} T t_{n} \\
t_{n}=\left(1-\beta_{n}\right) s_{n}+\beta_{n} T s_{n},
\end{gathered}
$$

where $\left\{\alpha_{n}\right\}$ and $\left\{\beta_{n}\right\}$ are sequences of positive numbers in $[0,1]$.

In 2000, M. A. Noor defined the Noor iterative scheme [4] as

$$
\begin{gathered}
p_{n+1}=\left(1-\alpha_{n}\right) p_{n}+\alpha_{n} T q_{n} \\
q_{n}=\left(1-\beta_{n}\right) p_{n}+\beta_{n} T r_{n} \\
r_{n}=\left(1-\gamma_{n}\right) p_{n}+\gamma_{n} T p_{n},
\end{gathered}
$$

where $\left\{\alpha_{n}\right\},\left\{\beta_{n}\right\}$ and $\left\{\gamma_{n}\right\}$ are sequences of positive numbers in $[0,1]$.

Recently, Phuengrattana and Suantai defined the SP iteration scheme [5] as 


$$
\begin{gathered}
x_{n+1}=\left(1-\alpha_{n}\right) y_{n}+\alpha_{n} T y_{n} \\
y_{n}=\left(1-\beta_{n}\right) z_{n}+\beta_{n} T z_{n} \\
z_{n}=\left(1-\gamma_{n}\right) x_{n}+\gamma_{n} T x_{n},
\end{gathered}
$$

where $\left\{\alpha_{n}\right\},\left\{\beta_{n}\right\}$ and $\left\{\gamma_{n}\right\}$ are sequences of positive numbers in $[0,1]$.

\section{Remarks:}

1) If $\gamma_{n}=0$, then the Noor iterative scheme (1.6) reduces to the Ishikawa iterative scheme (1.4).

2) If $\beta_{n}=\gamma_{n}=0$, then the Noor iterative scheme (1.6) reduces to the Mann iterative scheme (1.3).

3) In addition, when $\beta_{n}=\gamma_{n}=0$, then the SP iterative scheme (1.7) reduces to the Mann iterative scheme (1.3).

In 1972, Zamfirescu [6] obtained the following interesting fixed point theorem.

Theorem 1.1. Let $(X, d)$ be a complete metric space and $T: X \rightarrow X$ a mapping for which there exists real numbers $a, b$ and $c$ satisfying $a \in(0,1), b, c \in\left(0, \frac{1}{2}\right)$ such that for each pair $x, y \in X$, at least one of the following conditions hold

$$
\begin{aligned}
& \text { 1) } d(T x, T y) \leq a d(x, y) \\
& \text { 2) } d(T x, T y) \leq b[d(x, T x)+d(y, T y)] \\
& \text { 3) } d(T x, T y) \leq c[d(x, T y)+d(y, T x)] .
\end{aligned}
$$

Then $T$ has a fixed point $p$ and the Picard iteration $\left\{x_{n}\right\}_{n=0}^{\infty}$ defined by

$$
x_{n+1}=T x_{n}, n=0,1, \cdots
$$

converges to $p$ for any arbitrary but fixed $x_{0} \in X$.

The operators satisfying the condition (1.8) are called Zamfirescu operators.

Berinde [7] introduced a new class of operators on an arbitrary Banach space satisfying

$$
d(T x, T y) \leq 2 \delta d(x, T x)+\delta d(x, y),
$$

$\forall x, y \in X$ and some $\delta \in[0,1)$.

He proved that this class is wider than the class of Zamfirescu operators and used the Ishikawa iteration process to approximate fixed points of this class of operators in an arbitrary Banach space given in the form of following:

Theorem 1.2 [7]. Let $K$ be a nonempty closed convex subset of an arbitrary Banach space $X$ and $T: K \rightarrow K$ be a mapping satisfying (1.9). Let $\left\{s_{n}\right\}$ be defined through the Ishikawa iteration (1.4) and $s_{0} \in K$ where $\left\{\alpha_{n}\right\},\left\{\beta_{n}\right\}$ are sequences of positive numbers in $[0,1]$ with $\left\{\alpha_{n}\right\}$ satisfying $\sum_{n=0}^{\infty} \alpha_{n}=\infty$. Then $\left\{s_{n}\right\}$ converges strongly to the fixed point of $T$.
Several authors [5,8-17] have studied the equivalence between various iterative schemes. S. M. Solutz $[15,16]$ proved that for quasi-contractive operators the itérative processes Picard, Mann, Ishikawa and Noor are équivalent. Recently, Renu Chugh and Vivek Kumar [17] proved that for quasi-contractive operators satisfying (1.9) Picard, Mann, Ishikawa, Noor and SP iterative schemes are equivalent.

Fixed point iterative procedures are designed to be applied in solving equations arising in physical formulation but there is no systematic study of numerical aspects of these iterative procedures. In computational mathematics, it is of vital interest to know which of the given iterative procedures converge faster to a desired solution, commonly known as rate of convergence. B. E. Rhoades [18] compared the Mann and Ishikawa iterative procedures concerning their rate of convergence. He illustrated the difference in the rate of convergence for increasing and decreasing functions. Indeed he used computer programs, perhaps for the first time to compare the Mann and Ishikawa iterations through examples. S. L. Singh [19] extended the work of Rhoades. Very recently, Phuengrattana and Suantai [5] proved that SP iterative scheme is equivalent to and faster than Mann, Ishikawa and Noor iterative schemes for increasing functions.

Now, we introduce the following CR iterative process: Let $X$ be a Banach space, $T: X \rightarrow X$ a self map of $X$ and $x_{0} \in X$. Define the sequence $\left\{x_{n}\right\}_{n=0}^{\infty}$ by

$$
\begin{aligned}
& x_{n+1}=\left(1-\alpha_{n}\right) y_{n}+\alpha_{n} T y_{n} \\
& y_{n}=\left(1-\beta_{n}\right) T x_{n}+\beta_{n} T z_{n} \\
& z_{n}=\left(1-\gamma_{n}\right) x_{n}+\gamma_{n} T x_{n},
\end{aligned}
$$

where $\left\{\alpha_{n}\right\},\left\{\beta_{n}\right\}$ and $\left\{\gamma_{n}\right\}$ are sequences of positive numbers in $[0,1]$ with $\left\{\alpha_{n}\right\}$ satisfying $\sum_{n=0}^{\infty} \alpha_{n}=\infty$.

We shall need the following lemma and definitions.

Lemma 1 [20]. If $\delta$ is a real number such that $0 \leq \delta<1$ and $\left\{\epsilon_{n}\right\}_{n=0}^{\infty}$ is a sequence of positive numbers such that $\lim _{n \rightarrow \infty} \in_{n}=0$, then for any sequence of positive numbers $\left\{\epsilon_{n}\right\}_{n=0}^{\infty}$ satisfying

$$
u_{n+1} \leq \delta u_{n}+\in_{n}, \quad n=0,1,2, \cdots,
$$

we have $\lim _{n \rightarrow \infty} u_{n}=0$.

Definition 1.1. Suppose that $\left\{a_{n}\right\}$ and $\left\{b_{n}\right\}$ are two real convergent sequences with limits $a$ and $b$ respectively. Then $\left\{a_{n}\right\}$ is said to converge faster than $\left\{b_{n}\right\}$ if $\lim _{n \rightarrow \infty}\left|\frac{a_{n}-a}{b_{n}-b}\right|=0$. 
Definition 1.2 [21]. Suppose that $\left\{u_{n}\right\}$ and $\left\{v_{n}\right\}$ are two fixed point iteration procedures both converging to the same fixed point $p$ with the error estimates

$$
\begin{gathered}
\left\|u_{n}-p\right\| \leq a_{n}, n=0,1, \cdots \\
\left\|v_{n}-p\right\| \leq b_{n}, n=0,1, \cdots
\end{gathered}
$$

where $\left\{a_{n}\right\}$ and $\left\{b_{n}\right\}$ are two real convergent sequences converging to 0 . If $\left\{a_{n}\right\}$ converges faster than $\left\{b_{n}\right\}$, then we say $\left\{u_{n}\right\}$ converges faster than $v_{n}$ to $p$.

The purpose of this paper is to show the convergence of the CR iterative scheme and to prove equivalence between Picard, Mann, Ishikawa, Noor, Agarwal et al., SP and CR iterative schemes for quasi-contractive operators satisfying (1.9). We provide an example for which the $\mathrm{CR}$ iterative scheme is faster than the other above mentioned iterative schemes for the aforementioned class of operators. Also, by using computer programs in $\mathrm{C}++$, we compare the above mentioned iterative schemes through examples of increasing and decreasing functions.

\section{Result on Strong Convergence}

Theorem 2.1. Let $K$ be a nonempty closed convex subset of an arbitrary Banach space $X$ and $T: K \rightarrow K$ a mapping satisfying (1.9). Let $\left\{x_{n}\right\}_{n=0}^{\infty}$ be defined through the CR iteration (1.10) and $x_{0} \in X$, where $\left\{\alpha_{n}\right\},\left\{\beta_{n}\right\}$ are sequences of positive numbers in [0,1] with $\left\{\alpha_{n}\right\}$ Satisfying $\sum_{n=0}^{\infty} \alpha_{n}=\infty$. Then $\left\{x_{n}\right\}_{n=0}^{\infty}$ converges strongly to the fixed point of $T$.

Proof. Theorem 1.1 shows that $T$ has a unique fixed point in $K$, say $p$.

From (1.10), we have

$$
\begin{aligned}
& \left\|x_{n+1}-p\right\| \\
& \leq\left(1-\alpha_{n}\right)\left\|y_{n}-p\right\| \\
& +\alpha_{n}\left\|T y_{n}-p\right\| \\
& \leq\left(1-\alpha_{n}\right)\left(1-\beta_{n}\right)\left\|T x_{n}-p\right\| \\
& +\left(1-\alpha_{n}\right) \beta_{n}\left\|T z_{n}-p\right\| \\
& +\alpha_{n}\left\|T y_{n}-p\right\|,
\end{aligned}
$$

Using (1.9), (2.1) yields

$$
\begin{aligned}
& \left\|x_{n+1}-p\right\| \\
& \leq \delta\left(1-\alpha_{n}\right)\left(1-\beta_{n}\right)\left\|x_{n}-p\right\| \\
& +\delta\left(1-\alpha_{n}\right) \beta_{n}\left\|z_{n}-p\right\| \\
& +\delta \alpha_{n}\left\|y_{n}-p\right\|
\end{aligned}
$$

Using (1.9) and (1.10), (2.2) yields

$$
\begin{aligned}
& \left\|x_{n+1}-p\right\| \\
& \leq \delta\left(1-\alpha_{n}\right)\left(1-\beta_{n}\right)\left\|x_{n}-p\right\| \\
& +\delta\left(1-\alpha_{n}\right) \beta_{n}\left(1-\gamma_{n}\right)\left\|x_{n}-p\right\| \\
& +\delta\left(1-\alpha_{n}\right) \beta_{n} \gamma_{n}\left\|T x_{n}-p\right\| \\
& +\delta \alpha_{n}\left(1-\beta_{n}\right)\left\|T x_{n}-p\right\| \\
& +\delta \alpha_{n} \beta_{n}\left\|T z_{n}-p\right\| \\
& \leq \delta\left(1-\alpha_{n}\right)\left(1-\beta_{n}\right)\left\|x_{n}-p\right\| \\
& +\delta\left(1-\alpha_{n}\right) \beta_{n}\left(1-\gamma_{n}\right)\left\|x_{n}-p\right\| \\
& +\delta^{2}\left(1-\alpha_{n}\right) \beta_{n} \gamma_{n}\left\|x_{n}-p\right\| \\
& +\delta^{2} \alpha_{n}\left(1-\beta_{n}\right)\left\|x_{n}-p\right\| \\
& +\delta^{2} \alpha_{n} \beta_{n}\left(1-\gamma_{n}\right)\left\|x_{n}-p\right\| \\
& +\delta^{3} \alpha_{n} \beta_{n} \gamma_{n}\left\|x_{n}-p\right\| \\
& =\delta\left\{1-\alpha_{n}(1-\delta)-(1-\delta)\left(1-\alpha_{n}\right) \beta_{n} \gamma_{n}\right. \\
& \left.-\delta(1-\delta) \alpha_{n} \beta_{n} \gamma_{n}\right\}\left\|x_{n}-p\right\| \\
& \left\|x_{n}-p\right\| \leq \delta^{n} \prod_{k=0}^{n}\left[1-\alpha_{k}(1-\delta)\right]\left\|x_{0}-p\right\| \\
& \quad \leq \delta^{n} \mathrm{e} \\
& -(1-\delta) \sum_{k=0}^{\infty} \alpha_{k}\left\|x_{0}-p\right\|
\end{aligned}
$$

Since $0 \leq \delta<1, \alpha_{k} \in[0,1]$ and $\sum_{n=0}^{\infty} \alpha_{n}=\infty$, so $\delta^{n} \mathrm{e}^{-(1-\delta) \sum_{k=0}^{\infty} \alpha_{k}} \rightarrow 0$ as $n \rightarrow \infty$. Hence, it follows from (2.4) that $\lim _{n \rightarrow \infty}\left\|x_{n+1}-p\right\|=0$. Therefore $\left\{x_{n}\right\}_{n=0}^{\infty}$ converges strongly to $p$.

\section{Equivalence between Picard, Mann, Ishikawa, Noor, Agarwal et al., SP and CR Iterative Schemes}

Theorem 3.1. Let $K$ be a nonempty closed convex subset of a Banach space $X$ and $T: K \rightarrow K$ a mapping satisfying (1.9). If the initial point is the same, $\alpha_{n} \geq A>0, \forall n \in N$, then the followings are equivalent:

1) The Mann iteration (1.3) converges to $p$.

2) The Agarwal et al. iteration (1.5) converges to $p$.

3) The CR iteration (1.10) converges to $p$.

Proof. First we prove that 1$) \Leftrightarrow 3$ ). Let the Mann iteration (1.3) converge to $p$. We shall show that the CR iteration (1.10) also converges to $p$.

Using (1.3) and (1.10), we have

$$
\begin{aligned}
& \left\|x_{n+1}-u_{n+1}\right\| \\
& \leq\left(1-\alpha_{n}\right)\left\|y_{n}-u_{n}\right\|+\alpha_{n}\left\|T y_{n}-T u_{n}\right\| \\
& \leq\left(1-\alpha_{n}\right)\left\|y_{n}-u_{n}\right\|+\alpha_{n} \delta\left\|y_{n}-u_{n}\right\|+2 \alpha_{n} \delta\left\|u_{n}-T u_{n}\right\| \\
& \leq\left(1-\alpha_{n}(1-\delta)\right)\left\|y_{n}-u_{n}\right\|+2 \alpha_{n} \delta\left\|u_{n}-T u_{n}\right\| .
\end{aligned}
$$


From (1.10), we have

$$
\begin{aligned}
& \left\|y_{n}-u_{n}\right\| \\
& \leq\left(1-\beta_{n}\right)\left\|T x_{n}-u_{n}\right\|+\beta_{n}\left\|T z_{n}-u_{n}\right\| \\
& \leq\left(1-\beta_{n}\right)\left\|T x_{n}-T u_{n}\right\|+\left(1-\beta_{n}\right)\left\|T u_{n}-u_{n}\right\| \\
& \quad+\beta_{n}\left\{\left\|T z_{n}-T u_{n}\right\|+\left\|T u_{n}-u_{n}\right\|\right\} \\
& \leq \delta\left(1-\beta_{n}\right)\left\|x_{n}-u_{n}\right\|+2 \delta\left(1-\beta_{n}\right)\left\|T u_{n}-u_{n}\right\| \\
& \quad+\left(1-\beta_{n}\right)\left\|T u_{n}-u_{n}\right\|+\delta \beta_{n}\left\|z_{n}-u_{n}\right\| \\
& \quad+2 \delta \beta_{n}\left\|T u_{n}-u_{n}\right\|+\beta_{n}\left\|T u_{n}-u_{n}\right\| \\
& \leq \delta\left(1-\beta_{n}\right)\left\|x_{n}-u_{n}\right\|+(2 \delta+1)\left\|u_{n}-T u_{n}\right\| \\
& \quad+\delta \beta_{n}\left\|z_{n}-u_{n}\right\|,
\end{aligned}
$$

Again, from (1.10), we have

$$
\begin{aligned}
& \left\|z_{n}-u_{n}\right\| \\
& \leq\left(1-\gamma_{n}\right)\left\|x_{n}-u_{n}\right\|+\gamma_{n}\left\|T x_{n}-u_{n}\right\| \\
& \leq\left(1-\gamma_{n}\right)\left\|x_{n}-u_{n}\right\|+\gamma_{n}\left\{\left\|T x_{n}-T u_{n}\right\|+\left\|T u_{n}-u_{n}\right\|\right\} \\
& \leq\left(1-\gamma_{n}(1-\delta)\right)\left\|x_{n}-u_{n}\right\|+(2 \delta+1) \gamma_{n}\left\|T u_{n}-u_{n}\right\| .
\end{aligned}
$$

Substituting (3.3) in (3.2), we have

$$
\begin{aligned}
\left\|y_{n}-u_{n}\right\| \leq & \delta\left(1-\beta_{n}\right)\left\|x_{n}-u_{n}\right\| \\
& +\delta \beta_{n}\left(1-\gamma_{n}(1-\delta)\right)\left\|x_{n}-u_{n}\right\| \\
& +\left(\delta \beta_{n} \gamma_{n}+1\right)(2 \delta+1)\left\|T x_{n}-u_{n}\right\| .
\end{aligned}
$$

Substituting (3.4) in (3.1), we obtain

$$
\begin{aligned}
& \left\|x_{n+1}-u_{n+1}\right\| \\
& \leq\left(1-\alpha_{n}(1-\delta)\right)\left(\delta-\delta \beta_{n} \gamma_{n}(1-\delta)\right)\left\|x_{n}-u_{n}\right\| \\
& \quad+\left[\left(1-\alpha_{n}(1-\delta)\right)\left(\delta \beta_{n} \gamma_{n}+1\right)(2 \delta+1)\right. \\
& \left.\quad+2 \alpha_{n} \delta\right]\left\|u_{n}-T u_{n}\right\| .
\end{aligned}
$$

Also,

$$
\begin{aligned}
\left\|u_{n}-T u_{n}\right\| & \leq\left\|u_{n}-p\right\|+\left\|p-T u_{n}\right\| \\
& \leq(1+\delta)\left\|u_{n}-p\right\| .
\end{aligned}
$$

Substituting (3.6) in (3.5), we have

$$
\begin{aligned}
& \left\|x_{n+1}-u_{n+1}\right\| \\
& \leq\left(1-\alpha_{n}(1-\delta)\right)\left(\delta-\delta \beta_{n} \gamma_{n}(1-\delta)\right)\left\|x_{n}-u_{n}\right\| \\
& +(1+\delta)\left[\left(1-\alpha_{n}(1-\delta)\right)\left(\delta \beta_{n} \gamma_{n}+1\right)(2 \delta+1)+2 \alpha_{n} \delta\right] \\
& \cdot\left\|u_{n}-p\right\| \\
& \leq h\left\|x_{n}-u_{n}\right\|+\left\|u_{n}-p\right\|
\end{aligned}
$$

where $h=\left(1-\alpha_{n}(1-\delta)\right)\left(\delta-\delta \beta_{n} \gamma_{n}(1-\delta)\right)<1 \quad$ (using $\left.\alpha_{n} \geq A>0, \forall n \in N\right)$ and

$$
l=(1+\delta)[(1+\delta)(2 \delta+1)+2 \delta] .
$$

Using $u_{n} \rightarrow p$ as $n \rightarrow \infty$ and Lemma 1.1, (3.7) yields

$$
\left\|x_{n}-u_{n}\right\| \rightarrow 0 \text { as } n \rightarrow \infty .
$$

In addition

$$
\left\|x_{n}-p\right\| \leq\left\|x_{n}-u_{n}\right\|+\left\|u_{n}-p\right\|
$$

and this implies that $x_{n} \rightarrow p$ as $n \rightarrow \infty$.

Conversely, we prove that $x_{n} \rightarrow p$ implies $u_{n} \rightarrow p$.

Using (1.3), (1.9) and (1.10), we have

$$
\begin{aligned}
& \left\|x_{n+1}-u_{n+1}\right\| \\
& \leq\left(1-\alpha_{n}\right)\left\|y_{n}-u_{n}\right\|+\alpha_{n}\left\|T y_{n}-T u_{n}\right\| \\
& \leq\left(1-\alpha_{n}\right)\left\|y_{n}-u_{n}\right\|+\delta \alpha_{n}\left\|y_{n}-u_{n}\right\| \\
& +2 \delta \alpha_{n}\left\|y_{n}-T y_{n}\right\| \\
& \leq\left[1-\alpha_{n}(1-\delta)\right]\left\|y_{n}-u_{n}\right\|+2 \delta \alpha_{n}\left\|y_{n}-T y_{n}\right\| .
\end{aligned}
$$

From (1.10), we have

$$
\begin{aligned}
& \left\|y_{n}-u_{n}\right\| \\
& \leq\left(1-\beta_{n}\right)\left\|T x_{n}-u_{n}\right\|+\beta_{n}\left\|T z_{n}-u_{n}\right\| \\
& \leq\left(1-\beta_{n}\right)\left\|T x_{n}-x_{n}\right\|+\left(1-\beta_{n}\right)\left\|x_{n}-u_{n}\right\| \\
& \quad+\beta_{n}\left\{\left\|T z_{n}-x_{n}\right\|+\left\|x_{n}-u_{n}\right\|\right\} \\
& \leq\left(1-\beta_{n}\right)\left\|T x_{n}-p\right\|+\left(1-\beta_{n}\right)\left\|x_{n}-p\right\|+\left(1-\beta_{n}\right)\left\|x_{n}-u_{n}\right\| \\
& \quad+\beta_{n}\left\|T z_{n}-p\right\|+\beta_{n}\left\|x_{n}-p\right\|+\beta_{n}\left\|x_{n}-u_{n}\right\| \\
& \leq\left\|x_{n}-u_{n}\right\|+\left(\delta\left(1-\beta_{n}\right)+1\right)\left\|x_{n}-p\right\|+\delta \beta_{n}\left\|z_{n}-p\right\| \\
& \leq\left\|x_{n}-u_{n}\right\|+\left(\delta\left(1-\beta_{n}\right)+1\right)\left\|x_{n}-p\right\| \\
& \quad+\delta \beta_{n}\left(1-\gamma_{n}\right)\left\|x_{n}-p\right\| \\
& \quad+\delta^{2} \beta_{n} \gamma_{n}\left\|x_{n}-p\right\| \\
& \leq\left\|x_{n}-u_{n}\right\|+\left(1+\delta-\delta \beta_{n} \gamma_{n}(1-\delta)\right)\left\|x_{n}-p\right\| .
\end{aligned}
$$

Also,

$$
\begin{aligned}
& \left\|y_{n}-T y_{n}\right\| \\
& \leq\left\|y_{n}-p\right\|+\left\|T y_{n}-p\right\| \\
& \leq(1+\delta)\left\|y_{n}-p\right\| \\
& \leq \delta(1+\delta)\left(1-\beta_{n}\right)\left\|x_{n}-p\right\|+\delta(1+\delta) \beta_{n}\left\|z_{n}-p\right\| \\
& \leq \delta(1+\delta)\left\{\left(1-\beta_{n}\right)+\beta_{n}\left(1-\gamma_{n}\right)+\delta \beta_{n} \gamma_{n}\right\}\left\|x_{n}-p\right\| \\
& =\delta(1+\delta)\left\{1-\beta_{n} \gamma_{n}(1-\delta)\right\}\left\|x_{n}-p\right\|,
\end{aligned}
$$

Substituting (3.9) and (3.10) in (3.8) and rearranging the terms, we have 


$$
\begin{aligned}
\| & x_{n+1}-u_{n+1} \| \\
\leq & \left(1-\alpha_{n}(1-\delta)\right)\left\|x_{n}-u_{n}\right\| \\
& +\left\{( 1 - \alpha _ { n } ( 1 - \delta ) ) \left(1+\delta-\delta \beta_{n} \gamma_{n}((1-\delta))\right.\right. \\
& \left.+2 \alpha_{n} \delta^{2}(1+\delta)\left(1-\beta_{n} \gamma_{n}(1-\delta)\right)\right\}\left\|x_{n}-p\right\| \\
\leq & {\left[1-\alpha_{n}(1-\delta)\right]\left\|x_{n}-u_{n}\right\| } \\
& +\left(1+\delta+2 \delta^{2}(1+\delta)\right)\left\|x_{n}-p\right\| .
\end{aligned}
$$

Since $\alpha_{n} \geq A>0, \forall n \in N$, so $0 \leq 1-\alpha_{n}(1-\delta)<1, \forall n \in N$. Also $x_{n} \rightarrow p$ as $n \rightarrow \infty$. Hence, using Lemma 1.1, (3.11) yields $\left\|x_{n}-u_{n}\right\| \rightarrow 0$ as $n \rightarrow \infty$.

In addition $\left\|u_{n}-p\right\| \leq\left\|x_{n}-u_{n}\right\|+\left\|x_{n}-p\right\|$ and this implies that $u_{n} \rightarrow p$ as $n \rightarrow \infty$. Hence the result.

Next, we show that 1) $\Leftrightarrow 2$ ).

Let $u_{n} \rightarrow p$ as $n \rightarrow \infty$. Using (1.3) and (1.5), we have

$$
\begin{aligned}
& \left\|S_{n+1}-u_{n+1}\right\| \\
& \leq\left(1-\alpha_{n}\right)\left\|T s_{n}-u_{n}\right\|+\alpha_{n}\left\|T t_{n}-T u_{n}\right\| \\
& \leq\left(1-\alpha_{n}\right)\left\|T s_{n}-u_{n}\right\|+\delta \alpha_{n}\left\|t_{n}-u_{n}\right\|+2 \delta \alpha_{n}\left\|u_{n}-T u_{n}\right\| \\
& \leq\left(1-\alpha_{n}\left(1-\delta \beta_{n}\right)\right)\left\|T s_{n}-T u_{n}\right\| \\
& \quad+\left(1-\alpha_{n}\left(1-\delta \beta_{n}\right)\right)\left\|T u_{n}-u_{n}\right\| \\
& \quad+\delta \alpha_{n}\left(1-\beta_{n}\right)\left\|s_{n}-u_{n}\right\|+2 \delta \alpha_{n}\left\|u_{n}-T u_{n}\right\| .
\end{aligned}
$$

Now, we have the following estimates:

$$
\begin{aligned}
\left\|u_{n}-T u_{n}\right\| & \leq\left\|u_{n}-p\right\|+\left\|p-T u_{n}\right\| \\
& \leq(1+\delta)\left\|u_{n}-p\right\| \\
\left\|T s_{n}-T u_{n}\right\| & \leq \delta\left\|s_{n}-u_{n}\right\|+2 \delta\left\|u_{n}-T u_{n}\right\| \\
& \leq \delta\left\|s_{n}-u_{n}\right\|+2 \delta(1+\delta)\left\|u_{n}-p\right\| .
\end{aligned}
$$

It follows using (3.12), (3.13) and (3.14) that

$$
\begin{aligned}
& \left\|S_{n+1}-u_{n+1}\right\| \\
& \leq \delta\left\{\left(1-\alpha_{n}\left(1-\delta \beta_{n}\right)+\alpha_{n}\left(1-\beta_{n}\right)\right\}\left\|s_{n}-u_{n}\right\|\right. \\
& \quad+2 \delta\left(1-\alpha_{n}\left(1-\delta \beta_{n}\right)\right)(1+\delta)\left\|u_{n}-p\right\| \\
& \quad+\left(1-\alpha_{n}\left(1-\delta \beta_{n}\right)\right)(1+\delta)\left\|u_{n}-p\right\| \\
& \quad+2 \delta \alpha_{n}(1+\delta)\left\|u_{n}-p\right\| \\
& \leq \delta\left\{\left(1-\alpha_{n} \beta_{n}(1-\delta)\right)\right\}\left\|s_{n}-u_{n}\right\| . \\
& \quad+(1+4 \delta)(1+\delta)\left\|u_{n}-p\right\|
\end{aligned}
$$

Since $\delta \in[0,1)$ and $u_{n} \rightarrow p$ as $n \rightarrow \infty$, hence using Lemma 1.1, (3.15) yields $\left\|s_{n}-u_{n}\right\| \rightarrow 0$ as $n \rightarrow \infty$. In addition

$$
\left\|s_{n}-p\right\| \leq\left\|s_{n}-u_{n}\right\|+\left\|u_{n}-p\right\| .
$$

and this implies that $s_{n} \rightarrow p$ as $n \rightarrow \infty$.

Conversely, we prove that $s_{n} \rightarrow p$ implies $u_{n} \rightarrow p$. Using (1.3), (1.5) and (1.9), we have

$$
\begin{aligned}
& \left\|s_{n+1}-u_{n+1}\right\| \\
& \leq\left(1-\alpha_{n}\right)\left\|T s_{n}-u_{n}\right\|+\alpha_{n}\left\|T t_{n}-T u_{n}\right\| \\
& \leq\left(1-\alpha_{n}\right)\left\{\left\|T s_{n}-s_{n}\right\|+\left\|s_{n}-u_{n}\right\|\right\} \\
& \quad+\delta \alpha_{n}\left\|t_{n}-u_{n}\right\|+2 \delta \alpha_{n}\left\|t_{n}-T t_{n}\right\| .
\end{aligned}
$$

Now, we have the following estimates:

$$
\begin{aligned}
\left\|s_{n}-T s_{n}\right\| & \leq(1+\delta)\left\|s_{n}-p\right\| \\
\left\|t_{n}-T t_{n}\right\| & \leq(1+\delta)\left\|t_{n}-p\right\| \\
& \leq(1+\delta)\left(1-\beta_{n}(1-\delta)\right)\left\|s_{n}-p\right\| \\
\left\|t_{n}-u_{n}\right\| & \leq\left(1-\beta_{n}\right)\left\|s_{n}-u_{n}\right\|+\beta_{n}\left\|T s_{n}-u_{n}\right\| \\
& \leq\left(1-\beta_{n}\right)\left\|s_{n}-u_{n}\right\|+\beta_{n}\left\|T s_{n}-u_{n}\right\| \\
& +\beta_{n}\left\|s_{n}-u_{n}\right\| \\
& \leq\left\|s_{n}-u_{n}\right\|+\beta_{n}(1+\delta)\left\|s_{n}-p\right\| .
\end{aligned}
$$

It follows from (3.17), (3.18), (3.19) and (3.20) that

$$
\begin{aligned}
& \left\|s_{n+1}-u_{n+1}\right\| \\
& \leq\left(1-\alpha_{n}(1-\delta)\right)\left\|s_{n}-u_{n}\right\| \\
& \quad+(1+\delta)\left\{1-\alpha_{n}+2 \delta \alpha_{n}\left(1-\beta_{n}(1-\delta)\right)+\delta \alpha_{n} \beta_{n}\right\} \\
& \quad \cdot\left\|s_{n}-p\right\| \\
& \leq\left(1-\alpha_{n}(1-\delta)\right)\left\|s_{n}-u_{n}\right\|+(1+\delta)(1+3 \delta)\left\|s_{n}-p\right\| .
\end{aligned}
$$

If $\alpha_{n} \geq A>0, \forall n \in N$, then $0 \leq 1-a_{n}(1-d)<1$. Also, $s_{n} \rightarrow p$ as $n \rightarrow \infty$.

Hence, using Lemma 1.1, (3.21) yields $\left\|u_{n}-s_{n}\right\| \rightarrow 0$ as $n \rightarrow \infty$.

In addition

$$
\left\|u_{n}-p\right\| \leq\left\|u_{n}-s_{n}\right\|+\left\|s_{n}-p\right\|
$$

and this implies that $u_{n} \rightarrow p$ as $n \rightarrow \infty$. Hence the result.

Keeping in mind Soltuz's results $[15,16]$ as well as Chugh and Kumar's result [17], Theorem 3.1 leads to the following corollary:

Corollary 3.2. Let $K$ be a nonempty closed convex subset of a Banach space $X$ and $T: K \rightarrow K$ a mapping satisfying (1.9). If the initial point is the same, $\alpha_{n} \geq A>0, \forall n \in N$, then the followings are equivalent:

1) The Picard iteration (1.1) converges to the fixed point $p$ of $T$,

2) The Mann iteration (1.3) converges to $p$,

3) The Ishikawa iteration (1.4) converges to $p$, 
4) The Noor iteration (1.6) converges to $p$,

5) The Agarwal et al. iteration (1.5) converges to $p$,

6) The SP iteration (1.7) converges to $p$,

7) The CR iteration (1.10) converges to $p$.

\section{Results on Fastness of CR Iterative Scheme for Quasi-Contractive Operators}

In [21] Berinde showed that Picard iteration is faster than Mann iteration for quasi-contractive operators satisfying (1.9). In [22], Qing and Rhoades by taking example showed that Ishikawa iteration is faster than Mann iteration for a certain quasi-contractive operator. Ciric, Lee and Rafiq [23], by providing an example, showed that Noor iterative scheme can be faster than Mann and Ishikawa iterative schemes for some quasi-contractive operator. Recently, Nawab Hussian et al. [24] provide an example of a quasi-contractive operator for which the iterative scheme (1.5) due to Agarwal et al. is faster than Mann and Ishikawa iterative schemes.

Now, we show that the CR iteration is faster than Picard, Agarwal et al., Noor and SP iterations for quasicontractive operators satisfying (1.9) as follows:

1) By providing an example 4.1 of a quasi-contractive operator satisfying (1.9), we show that CR iterative scheme is faster than Agarwal et al., Noor and SP iterative schemes.

2) By using definition (1.1), we show that CR iterative scheme is faster than Picard iteration.

1) Example 4.1. Let $T: T:[0,1] \rightarrow[0,1]$ be defined by $T(x)=\frac{x}{2}, \alpha_{n}=\beta_{n}=\gamma_{n}=0, n=1,2, \cdots, 15$, $\alpha_{n}=\beta_{n}=\gamma_{n}=\frac{4}{\sqrt{n}}, n \geq 16$. It is clear that $T$ is a quasicontractive operator satisfying (1.9) with a unique fixed point 0 . Also, it is easy to see that $T, \alpha_{n}, \beta_{n}$ and $\gamma_{n}$ satisfies all the conditions of Theorem 2.1. We show that CR iterative scheme is faster than Agarwal et al., Noor and SP iterative schemes.

Proof. First of all we show that CR iterative scheme is faster than Noor iterative scheme.

Let $n \geq 16$ and $p_{0}=x_{0}$. Then, from [23], for Noor iteration (1.6), we have

$$
p_{n+1}=\prod_{i=16}^{n}\left(1-\frac{2}{\sqrt{i}}-\frac{4}{i}-\frac{8}{i \sqrt{i}}\right) p_{0} .
$$

Also, for CR iteration (1.10), we have

$$
x_{n+1}=\prod_{i=16}^{n}\left(\frac{1}{2}-\frac{1}{\sqrt{i}}-\frac{4}{i}+\frac{8}{i \sqrt{i}}\right) x_{0} .
$$

So,

$$
\begin{aligned}
\frac{x_{n+1}}{p_{n+1}}= & \frac{\prod_{i=16}^{n}\left(\frac{1}{2}-\frac{1}{\sqrt{i}}-\frac{4}{i}+\frac{8}{i \sqrt{i}}\right) x_{0}}{\prod_{i=16}^{n}\left(1-\frac{2}{\sqrt{i}}-\frac{4}{i}-\frac{8}{i \sqrt{i}}\right) p_{0}} \\
\leq & \prod_{i=16}^{n}\left[1-\frac{\left(i^{\frac{3}{2}}-2 i-32\right)}{\left(2 i^{\frac{3}{2}}-8 \sqrt{i}-4 i-16\right)}\right] .
\end{aligned}
$$

It is easy to see that

$$
\begin{aligned}
0 & \leq \lim _{n \rightarrow \infty} \prod_{i=16}^{n}\left[1-\frac{\left(i^{\frac{3}{2}}-2 i-32\right)}{\left(2 i^{\frac{3}{2}}-8 \sqrt{i}-4 i-16\right)}\right] \\
& \leq \lim _{n \rightarrow \infty} \prod_{i=16}^{n}\left(1-\frac{1}{i}\right)=\lim _{n \rightarrow \infty} \frac{15}{n}=0 .
\end{aligned}
$$

Hence, we have $\lim _{n \rightarrow \infty}\left|\frac{x_{n+1}}{p_{n+1}}\right|=0$.

Therefore, by definition 1.2, CR iterative scheme converges faster than Noor iterative scheme to the fixed point 0 of $T$.

Secondly, we show that CR iterative scheme is faster than SP iterative scheme:

For SP iteration (1.7) we have

$$
x_{n+1}=\prod_{i=16}^{n}\left(1-\frac{6}{\sqrt{i}}+\frac{12}{i}-\frac{8}{i \sqrt{i}}\right) x_{0} .
$$

So,

$$
\begin{aligned}
\frac{x_{n+1}(\mathrm{CR})}{x_{n+1}(\mathrm{SP})} & =\frac{\prod_{i=16}^{n}\left(\frac{1}{2}-\frac{1}{\sqrt{i}}-\frac{4}{i}+\frac{8}{i \sqrt{i}}\right)}{\prod_{i=16}^{n}\left(1-\frac{6}{\sqrt{i}}+\frac{12}{i}-\frac{8}{i \sqrt{i}}\right)} \\
& =\prod_{i=16}^{n}\left[1-\frac{\left(\frac{1}{2}-\frac{5}{\sqrt{i}}+\frac{16}{i}-\frac{16}{i \sqrt{i}}\right)}{\left.1-\frac{6}{\sqrt{i}}+\frac{12}{i}-\frac{8}{i \sqrt{i}}\right]}\right] \\
& =\prod_{i=16}^{n}\left[1-\frac{\left(i^{\frac{3}{2}}-10 i+32 \sqrt{i}-32\right)}{2 i^{\frac{3}{2}}+24 \sqrt{i}-12 i-16}\right] .
\end{aligned}
$$

It is easy to see that 


$$
\begin{aligned}
& 0 \leq \lim _{n \rightarrow \infty} \prod_{i=16}^{n}\left[1-\frac{\left(i^{\frac{3}{2}}-10 i+32 \sqrt{i}-32\right)}{\left(2 i^{\frac{3}{2}}+24 \sqrt{i}-12 i-16\right)}\right] \\
& \leq \lim _{n \rightarrow \infty} \prod_{i=16}^{n}\left(1-\frac{1}{1000+i}\right)=\lim _{n \rightarrow \infty} \frac{1015}{n}=0 .
\end{aligned}
$$

Hence, we have $\lim _{n \rightarrow \infty}\left|\frac{x_{n+1}(\mathrm{CR})}{x_{n+1}(\mathrm{SP})}\right|=0$. Therefore, by definition 1.2, CR iterative scheme converges faster than SP iterative scheme to the fixed point 0 of $T$.

Next, we show that CR iterative scheme is faster than Agarwal et al. iterative scheme.

For Agarwal et al. iteration (1.5), we have

$$
s_{n+0 ?}=\prod_{i=16}^{n}\left(\frac{1}{2}-\frac{4}{i}\right) s \text {. }
$$

So,

$$
\begin{aligned}
\frac{x_{n+1}}{s_{n+1}} & =\frac{\prod_{i=16}^{n}\left(\frac{1}{2}-\frac{1}{\sqrt{i}}-\frac{4}{i}+\frac{8}{i \sqrt{i}}\right) x_{0}}{\prod_{i=16}^{n}\left(\frac{1}{2}-\frac{4}{i}\right) s_{0}} \\
& =\prod_{i=16}^{n}\left[1-\frac{\left(\frac{1}{\sqrt{i}}-\frac{8}{i \sqrt{i}}\right)}{\frac{1}{2}-\frac{4}{i}}\right] .
\end{aligned}
$$

It is easy to see that

$$
\begin{aligned}
0 & \leq \lim _{n \rightarrow \infty} \prod_{i=16}^{n}\left[1-\frac{\left(\frac{1}{\sqrt{i}}-\frac{8}{i \sqrt{i}}\right)}{\frac{1}{2}-\frac{4}{i}}\right] \leq \lim _{n \rightarrow \infty} \prod_{i=16}^{n}\left[1-\frac{1}{i}\right] \\
& =\lim _{n \rightarrow \infty} \frac{15}{n}=0 .
\end{aligned}
$$

Hence, we have $\lim _{n \rightarrow \infty}\left|\frac{x_{n+1}}{s_{n+1}}\right|=0$. Therefore, again by definition 1.2, CR iterative scheme converges faster than Agarwal et al. iterative scheme to the fixed point 0 of $T$.

2) Here we show that CR iteration is faster than Picard iteration.

Using Picard iteration (1.1) and condition (1.9) we have

$$
\left\|x_{n+1}-p\right\| \leq \delta^{n+1}\left\|x_{0}-p\right\|
$$

Also, from (2.3) we have

$$
\begin{aligned}
\left\|x_{n+1}-p\right\| \leq & \delta\left\{1-\alpha_{n}(1-\delta)-(1-\delta)\left(1-\alpha_{n}\right) \beta_{n} \gamma_{n}\right. \\
& \left.-\delta(1-\delta) \alpha_{n} \beta_{n} \gamma_{n}\right\}\left\|x_{n}-p\right\| \\
\leq & \delta q\left\|x_{n}-p\right\| \leq \delta^{n+1} q^{n+1}\left\|x_{0}-p\right\|,
\end{aligned}
$$

where

$$
\begin{aligned}
q=\left\{1-\alpha_{n}(1-\delta)-(1-\delta)\left(1-\alpha_{n}\right) \beta_{n} \gamma_{n}\right. \\
\left.-\delta(1-\delta) \alpha_{n} \beta_{n} \gamma_{n}\right\}<1
\end{aligned}
$$

(if $a_{n}>0, \forall n \in N$ ).

In order to compare $\mathrm{CR}$ and Picard iterations, we must compare the coefficients of the inequalities (4.4) and (4.3).

Obviously $\frac{\delta^{n+1} q^{n+1}}{\delta^{n+1}} \rightarrow 0$ as $n \rightarrow \infty$ and hence using definition (1.1), we can say that CR iteration is faster than Picard iteration.

Keeping in mind results of example 4.1 as well as Ciric et al.'s results [23], we conclude that CR iterative scheme is faster than other iterative schemes for a certain class of quasi-contractive operators.

\section{Applications}

In this section, with the help of computer programs in $\mathrm{C}++$, we compare the rate of convergence of Picard, Mann, Ishikawa, Noor, Agarwal et al., SP and CR iteration procedures, through examples. The outcome is listed in the form of Tables 1-4, by taking initial approximation $x_{0}=0.8$ and $a_{n}=b_{n}=g_{n}=\frac{1}{(1+n)^{\frac{1}{2}}}$, for all iterative schemes.

\subsection{Example of Decreasing Function}

Let $f:[0,1] \rightarrow[0,1]$ be defined by $f(x)=(1-x)^{m}$, $m=7,8, \cdots$. Then $f$ is a decreasing function. By taking $m=7$, the comparison of convergence of above mentioned iterative schemes to the exact fixed point $p=$ 0.188348 is listed in the Table $\mathbf{1}$.

\subsection{Example of Increasing Function}

Let $f:[0,8] \rightarrow[0,8]$ be defined as $f(x)=\frac{x^{2}+9}{10}$. Then $f$ is an increasing function. The comparison of convergence of above mentioned iterative schemes to the exact fixed point $p=1$ is listed in the Table 2 .

\subsection{Example of Cubic Equation}

To find solution of cubic equation $x^{3}+x^{2}-1=0$ means to find fixed point of the function $\left(1-x^{3}\right)^{1 / 2}$ as $x^{3}+x^{2}$ 


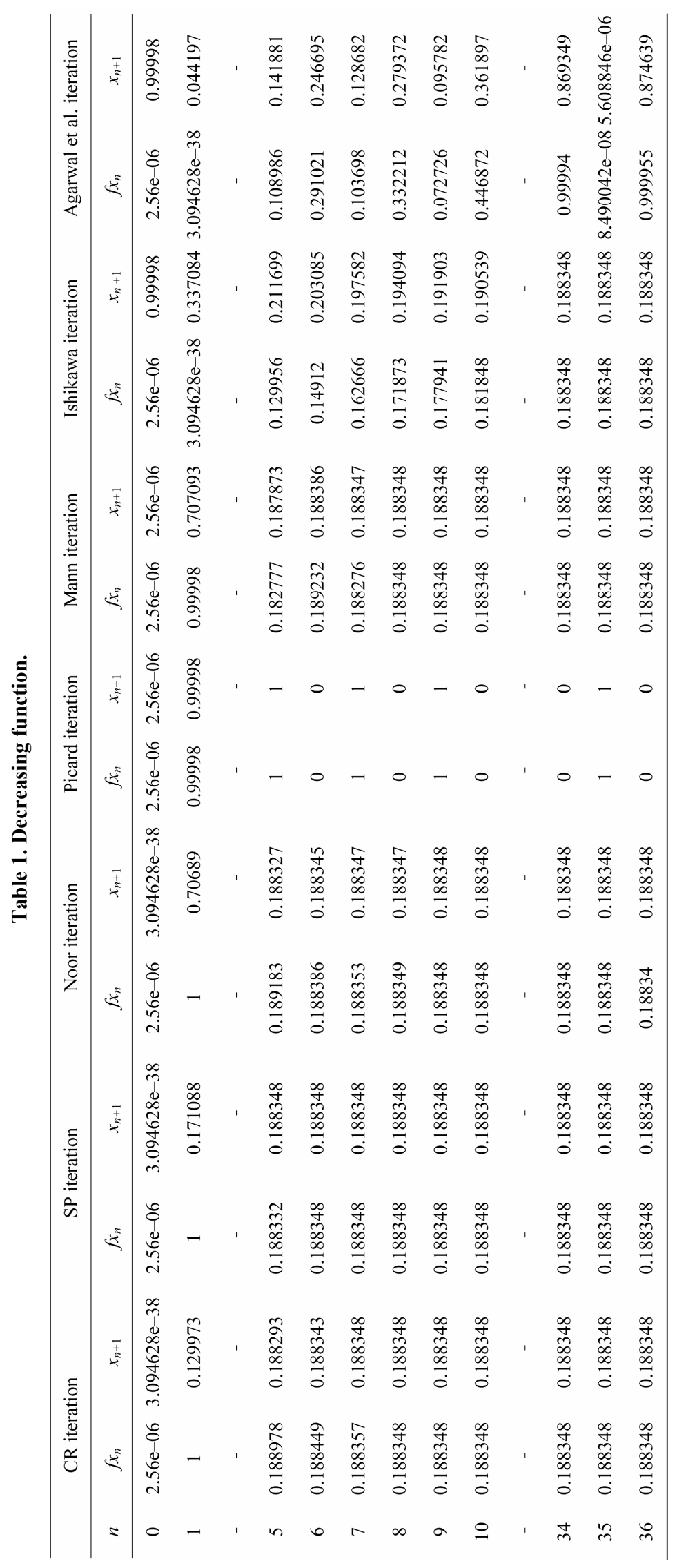


Table 2. Increasing function.

\begin{tabular}{|c|c|c|c|c|c|c|c|c|c|c|c|c|c|c|}
\hline \multicolumn{3}{|c|}{ CR iteration } & \multicolumn{2}{|c|}{ SP iteration } & \multicolumn{2}{|c|}{ Noor iteration } & \multicolumn{2}{|c|}{ Picard iteration } & \multicolumn{2}{|c|}{ Mann iteration } & \multicolumn{2}{|c|}{ Ishikawa iteration } & \multicolumn{2}{|c|}{$\begin{array}{c}\text { Agarwal et al. } \\
\text { iteration }\end{array}$} \\
\hline$n$ & $f x_{n}$ & $x_{n+1}$ & $f x_{n}$ & $x_{n+1}$ & $f x_{n}$ & $x_{n+1}$ & $n$ & $f x_{n}$ & $x_{n+1}$ & $f x_{n}$ & $x_{n+1}$ & $n$ & $x_{n+1}$ & $f x_{n}$ \\
\hline 0 & 0.964 & 0.998591 & 0.964 & 0.998591 & 0.964 & 0.998591 & 0.9 & 0.9 & 0.964 & 0.964 & 0.964 & 0.99293 & 0.964 & 0.99293 \\
\hline- & - & - & - & - & - & - & - & - & - & - & - & - & - & - \\
\hline 4 & 1 & 1 & 0.999999 & 0.999999 & 0.999974 & 0.999922 & 0.99985 & 0.99985 & 0.994714 & 0.982828 & 0.999968 & 0.999937 & 0.999996 & 0.999997 \\
\hline 5 & 1 & 1 & 1 & 1 & 0.999984 & 0.99995 & 0.99997 & 0.99997 & 0.996595 & 0.988449 & 0.999987 & 0.999973 & 0.999999 & 0.999999 \\
\hline 6 & 1 & 1 & 1 & 1 & 0.99999 & 0.999966 & 0.999994 & 0.999994 & 0.997703 & 0.991947 & 0.999968 & 0.999937 & 1 & 1 \\
\hline 7 & 1 & 1 & 1 & 1 & 0.999993 & 0.999976 & 0.999999 & 0.999999 & 0.998396 & 0.994227 & 0.999995 & 0.999988 & 1 & 1 \\
\hline 8 & 1 & 1 & 1 & 1 & 0.999995 & 0.999983 & 1 & 1 & 0.998849 & 0.995767 & 0.999998 & 0.999994 & 1 & 1 \\
\hline 23 & 1 & 1 & 1 & 1 & 1 & 1 & 1 & 1 & 0.999964 & 0.99985 & 0.999999 & 0.999997 & 1 & 1 \\
\hline 24 & 1 & 1 & 1 & 1 & 1 & 1 & 1 & 1 & 0.99997 & 0.999874 & 0.999999 & 0.999998 & 1 & 1 \\
\hline- & - & - & - & - & - & - & - & - & - & - & - & - & - & - \\
\hline 33 & 1 & 1 & 1 & 1 & 1 & 1 & 1 & 1 & 0.999994 & 0.999974 & 1 & 1 & 1 & 1 \\
\hline- & - & - & - & - & - & - & - & - & - & - & - & - & - & - \\
\hline 68 & 1 & 1 & 1 & 1 & 1 & 1 & 1 & 1 & 1 & 1 & 1 & 1 & 1 & 1 \\
\hline 69 & 1 & 1 & 1 & 1 & 1 & 1 & 1 & 1 & 1 & 1 & 1 & 1 & 1 & 1 \\
\hline
\end{tabular}

$-1=0$ can be rewritten as $\left(1-x^{3}\right)^{1 / 2}=x$. The comparison of convergence of above mentioned iterative schemes to the exact fixed point $p=0.754878$ of $\left(1-x^{3}\right)^{1 / 2}$ is listed in the Table 3 .

\subsection{Example of Goat Problem}

A farmer has a fenced circular pasture of radius $a$ and wants to tie a goat to the fence with a rope of length $b$ so as to allow the goat to graze half the pasture. How long should the rope be to accomplish this?

The length of the rope " $b$ " must be longer than " $a$ " and shorter than $\sqrt{2} a$, i.e. $a<b<\sqrt{2} a$.

If we let $x=\frac{b}{a}$, we get the simplified equation $\left(4-2 x^{2}\right) \sin ^{-1}\left(\frac{x}{2}\right)-x \sqrt{4-x^{2}}+\pi x^{2}=\pi$ and we are looking for the solution $x$, with $1<x<\sqrt{2}$. If we rearrange the equation, we can produce a sequence that will converge to the solution:

$$
\begin{gathered}
\pi x^{2}=\pi+x \sqrt{4-x^{2}}-\left(4-2 x^{2}\right) \sin ^{-1}\left(\frac{x}{2}\right) \\
\text { or } x=\sqrt{\frac{\pi+x \sqrt{4-x^{2}}-\left(4-2 x^{2}\right) \sin ^{-1}\left(\frac{x}{2}\right)}{\pi}} .
\end{gathered}
$$

Let $x_{1}=1$,

$$
x_{n+1}=\sqrt{\frac{\pi+x_{n} \sqrt{4-x_{n}^{2}}-\left(4-2 x_{n}^{2}\right) \sin ^{-1}\left(\frac{x_{n}}{2}\right)}{\pi}}
$$

and $f(x)=\sqrt{\frac{\pi+x_{n} \sqrt{4-x_{n}^{2}}-\left(4-2 x_{n}^{2}\right) \sin ^{-1}\left(\frac{x_{n}}{2}\right)}{\pi}}$.

The comparison of convergence of above mentioned iterative schemes to the exact fixed point 1.15863 of the function $f(x)$ is listed in the Table 4 . So the rope length " $b$ " should be approximately $1.15863 a$.

For detailed study, these programs are again executed after changing the parameters and the readings are recorded (discussed in the next section).

\section{Observations}

\subsection{Decreasing Function $(1-\boldsymbol{x})^{\boldsymbol{m}}$}

1) For $m=8$ and $x_{0}=0.8$, the Picard scheme never converges (oscillates between 0 and 1), the Mann scheme converges in 9 iterations, the Ishikawa scheme converges in 35 iterations, the Noor scheme converges in 10 iterations, Agarwal et al. iteration does not converges, the CR scheme converges in 9 iterations and the SP scheme converges in 7 iterations.

2) For $m=30$ and $x_{0}=0.8$, the Picard scheme never 
Table 3. Cubic equation.

\begin{tabular}{|c|c|c|c|c|c|c|c|c|c|c|c|c|c|c|}
\hline \multicolumn{3}{|c|}{ CR iteration } & \multicolumn{2}{|c|}{ SP iteration } & \multicolumn{2}{|c|}{ Noor iteration } & \multicolumn{2}{|c|}{ Picard iteration } & \multicolumn{2}{|c|}{ Mann iteration } & \multicolumn{2}{|c|}{ Ishikawa iteration } & \multicolumn{2}{|c|}{$\begin{array}{c}\text { Agarwal et al. } \\
\text { iteration }\end{array}$} \\
\hline$n$ & $f x_{n}$ & $x_{n+1}$ & $f x_{n}$ & $x_{n+1}$ & $f x_{n}$ & $x_{n+1}$ & $f x_{n}$ & $x_{n+1}$ & $f x_{n}$ & $x_{n+1}$ & $f x_{n}$ & $x_{n+1}$ & $f x_{n}$ & $x_{n+1}$ \\
\hline 0 & 0.69857 & 0.68185 & 0.69857 & 0.681845 & 0.69857 & 0.681845 & 0.69857 & 0.69857 & 0.69857 & 0.69857 & 0.69857 & 0.811848 & 0.69857 & 0.811848 \\
\hline- & - & - & - & - & - & - & - & - & - & - & - & - & - & - \\
\hline 3 & 0.754709 & 0.754883 & 0.754992 & 0.754878 & 0.754385 & 0.754963 & 0.826439 & 0.826439 & 0.76192 & 0.755248 & 0.723666 & 0.769363 & 0.756614 & 0.75569 \\
\hline 4 & 0.754872 & 0.754878 & 0.754878 & 0.754878 & 0.754782 & 0.754902 & 0.659955 & 0.659955 & 0.754458 & 0.754895 & 0.737969 & 0.762659 & 0.753957 & 0.75435 \\
\hline 5 & 0.754878 & 0.754878 & 0.754878 & 0.754878 & 0.75485 & 0.754886 & 0.844134 & 0.844134 & 0.754858 & 0.75488 & 0.745922 & 0.759043 & 0.755475 & 0.755263 \\
\hline - & - & - & - & - & - & - & - & - & - & - & - & - & - & - \\
\hline 8 & 0.754878 & 0.754878 & 0.754878 & 0.754878 & 0.754876 & 0.754878 & 0.593724 & 0.593724 & 0.754878 & 0.754878 & 0.753476 & 0.755566 & 0.754592 & 0.75466 \\
\hline 9 & 0.754878 & 0.754878 & 0.754878 & 0.754878 & 0.754877 & 0.754878 & 0.889217 & 0.889217 & 0.754878 & 0.754878 & 0.754097 & 0.755268 & 0.755124 & 0.755071 \\
\hline
\end{tabular}

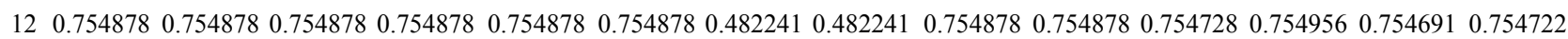

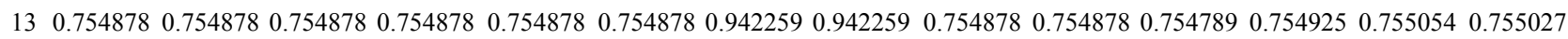

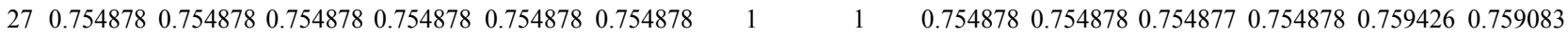

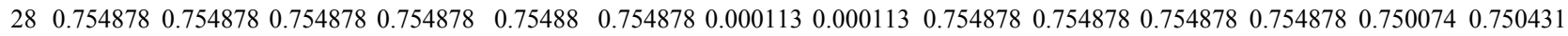

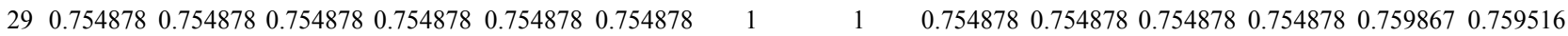

converges (oscillates between 0 and 1), the Mann scheme converges in 11 iterations, the Ishikawa scheme converges in 37 iterations, the Noor scheme converges in 12 iterations, Agarwal et al. scheme never converges, the CR scheme converges in 13 iterations and the SP scheme converges in 9 iterations.

3) Taking initial guess $x_{0}=0.2$ (nearer to the fixed point), Picard scheme never converges (oscillates between 0 and 1), Mann scheme converges in 10 iterations, Ishikawa scheme converges in 40 iterations, Noor scheme converges in 10 iterations, Agarwal et al. scheme does not converge, the CR scheme converges in 10 iterations and the SP scheme converges in 8 iterations.

4) Taking $\alpha_{n}=\beta_{n}=\gamma_{n}=\frac{1}{(1+n)^{\frac{1}{4}}}$ and $x_{0}=0.8$, we obtain that the Mann scheme converges in 23 iterations, the Ishikawa scheme converges in 56 iteration, the Noor scheme converges in 21 iterations, Agarwal et al. scheme converges in 12 iterations, the $\mathrm{CR}$ scheme converges in 11 iterations and the SP scheme converges in 15 iterations.

\subsection{Increasing Function $\left(x^{2}+9\right) / 10$}

1) For $x_{0}=0.8$, the Picard scheme converges to a fixed point in 8 iterations, the Mann scheme converges in 69 iterations, the Ishikawa scheme converges in 34 iterations, the Noor scheme converges in 24 iterations, Agarwal et al. scheme converges in 7 iterations, the SP scheme converges in 6 iterations and the CR scheme converges in 5 iterations.

2) Taking initial guess $x_{0}=0.6$ (away from the fixed point), the Picard scheme converges to a fixed point in 8 iterations, the Mann scheme converges in 75 iterations, the Ishikawa scheme converges in 38 iterations, the Noor scheme converges in 27 iterations, Agarwal et al. scheme converges in 6 iterations, the SP scheme converges in 7 iterations and the $\mathrm{CR}$ scheme converges in 5 iterations.

3) Taking, $\alpha_{n}=\beta_{n}=\gamma_{n}=\frac{1}{(1+n)^{\frac{1}{4}}}, x_{0}=0.8$, we ob-

$$
(1+n)^{\frac{1}{4}}
$$

tain that the Mann scheme converges in 23 iterations, the Ishikawa scheme converges in 12 iterations, Noor scheme converges in 9 iterations, Agarwal et al. scheme converges in 6 iterations, the SP scheme converges in 5 iterations and the CR scheme converges in 4 iterations.

\subsection{Cubic Equation $x^{3}+x^{2}-1=0$}

1) For $x_{0}=0.8$, the Picard scheme never converges to the solution of cubic equation (oscillates between 0 and 1), the Mann scheme converges in 9 iterations, the Ishikawa scheme converges in 29 iterations, the Noor sche- 
Table 4. Goat problem.

\begin{tabular}{|c|c|c|c|c|c|c|c|c|c|c|c|c|c|c|}
\hline \multicolumn{3}{|c|}{ CR iteration } & \multicolumn{2}{|c|}{ SP iteration } & \multicolumn{2}{|c|}{ Noor iteration } & \multicolumn{2}{|c|}{ Picard iteration } & \multicolumn{2}{|c|}{ Mann iteration } & \multicolumn{2}{|c|}{ Ishikawa iteration } & \multicolumn{2}{|c|}{$\begin{array}{l}\text { Agarwal et al. } \\
\text { iteration }\end{array}$} \\
\hline $\mathrm{n}$ & $f x_{n}$ & $x_{n+1}$ & $f x_{n}$ & $x_{n+1}$ & $f x_{n}$ & $x_{n+1}$ & $f x_{n}$ & $x_{n+1}$ & $f x_{n}$ & $x_{n+1}$ & $f x_{n}$ & $x_{n+1}$ & $f x_{n}$ & $x_{n+1}$ \\
\hline 0 & 1.103589 & 91.150601 & 1.103589 & 1.150601 & 1.103589 & 1.150601 & 1.103589 & 1.103589 & 1.103589 & 1.103589 & 1.103589 & 1.137884 & 1.103589 & 1.137884 \\
\hline 1 & 1.155492 & 21.157384 & 1.103589 & 1.150601 & 1.155492 & 1.155275 & 1.137884 & 1.137884 & 1.137884 & 1.137884 & 1.150601 & 1.149315 & 1.150601 & 1.15304 \\
\hline- & - & - & - & - & - & - & - & - & - & - & - & - & - & - \\
\hline 6 & 1.158629 & 91.158629 & 1.15861 & 1.158608 & 1.158414 & 1.158229 & 1.15844 & 1.15844 & 1.155693 & 1.152847 & 1.157988 & 1.157426 & 1.158603 & 1.158608 \\
\hline 7 & 1.15863 & 1.15863 & 1.158622 & 1.15862 & 1.158472 & 1.158328 & 1.158556 & 1.158556 & 1.156366 & 1.154091 & 1.158157 & 1.15772 & 1.15862 & 1.158621 \\
\hline 8 & 1.15863 & 1.15863 & 1.158626 & 1.158625 & 1.158512 & 1.158399 & 1.158601 & 1.158601 & 1.156852 & 1.155011 & 1.158273 & 1.157929 & 1.158627 & 1.158627 \\
\hline 9 & 1.15863 & 1.15863 & 1.158628 & 1.158628 & 1.158539 & 1.158449 & 1.158619 & 1.158619 & 1.157211 & 1.155707 & 1.158355 & 1.15808 & 1.158629 & 1.158629 \\
\hline 10 & 1.15863 & 1.15863 & 1.15863 & 1.15863 & 1.158559 & 1.158487 & 1.158626 & 1.158626 & 1.157484 & 1.156243 & 1.158414 & 1.158193 & 1.15863 & 1.15863 \\
\hline 11 & 1.15863 & 1.15863 & 1.15863 & 1.15863 & 1.158574 & 1.158515 & 1.158629 & 1.158629 & 1.157693 & 1.156661 & 1.158458 & 1.158278 & 1.15863 & 1.15863 \\
\hline 12 & 1.15863 & 1.15863 & 1.15863 & 1.15863 & 1.158585 & 1.158537 & 1.15863 & 1.15863 & 1.157857 & 1.156993 & 1.158492 & 1.158344 & 1.15863 & 1.15863 \\
\hline 13 & 1.15863 & 1.15863 & 1.15863 & 1.15863 & 1.158594 & 1.158554 & 1.15863 & 1.15863 & 1.157987 & 1.157259 & 1.158518 & 1.158395 & 1.15863 & 1.15863 \\
\hline 14 & 1.15863 & 1.15863 & 1.15863 & 1.15863 & 1.1586 & 1.158567 & 1.15863 & 1.15863 & 1.158092 & 1.157474 & 1.158538 & 1.158436 & 1.15863 & 1.15863 \\
\hline 15 & 1.15863 & 1.15863 & 1.15863 & 1.15863 & 1.158606 & 1.158578 & 1.15863 & 1.15863 & 1.158176 & 1.157649 & 1.158554 & 1.158468 & 1.15863 & 1.15863 \\
\hline 16 & 1.15863 & 1.15863 & 1.15863 & 1.15863 & 1.15861 & 1.158586 & 1.15863 & 1.15863 & 1.158245 & 1.157794 & 1.158567 & 1.158494 & 1.15863 & 1.15863 \\
\hline 17 & 1.15863 & 1.15863 & 1.15863 & 1.15863 & 1.158613 & 1.158593 & 1.15863 & 1.15863 & 1.158302 & 1.157914 & 1.158577 & 1.158516 & 1.15863 & 1.15863 \\
\hline 18 & 1.15863 & 1.15863 & 1.15863 & 1.15863 & 1.158616 & 1.158599 & 1.15863 & 1.15863 & 1.158349 & 1.158013 & 1.158585 & 1.158533 & 1.15863 & 1.15863 \\
\hline- & - & - & - & - & - & - & - & - & - & - & - & - & - & - \\
\hline 47 & 1.15863 & 1.15863 & 1.15863 & 1.15863 & 1.15863 & 1.15863 & 1.15863 & 1.15863 & 1.15862 & 1.158607 & 1.158629 & 1.158627 & 1.15863 & 1.15863 \\
\hline 48 & 1.15863 & 1.15863 & 1.15863 & 1.15863 & 1.15863 & 1.15863 & 1.15863 & 1.15863 & 1.158621 & 1.158609 & 1.158629 & 1.158628 & 1.15863 & 1.15863 \\
\hline- & - & - & - & - & - & - & - & - & -- & - & - & - & - & - \\
\hline 60 & 1.15863 & 1.15863 & 1.15863 & 1.15863 & 1.15863 & 1.15863 & 1.15863 & 1.15863 & 1.158627 & 1.158623 & 1.15863 & 1.15863 & 1.15863 & 1.15863 \\
\hline- & - & - & - & - & - & - & - & - & - & - & - & - & - & - \\
\hline 89 & 1.15863 & 1.15863 & 1.15863 & 1.15863 & 1.15863 & 1.15863 & 1.15863 & 1.15863 & 1.15863 & 1.15863 & 1.15863 & 1.15863 & 1.15863 & 1.15863 \\
\hline 90 & 1.15863 & 1.15863 & 1.15863 & 1.15863 & 1.15863 & 1.15863 & 1.15863 & 1.15863 & 1.15863 & 1.15863 & 1.15863 & 1.15863 & 1.15863 & 1.15863 \\
\hline
\end{tabular}

me converges in 13 iterations, Agarwal et al. scheme never converges, the $\mathrm{CR}$ scheme converges in 6 iterations and the SP scheme converges in 5 iterations.

2) Taking initial guess $x_{0}=0.1$ (away from the solution of cubic equation), Picard scheme never converges (oscillates between 0 and 1), the Mann scheme converges in 10 iterations, the Ishikawa scheme converges in 30 iterations, Noor scheme converges in 21 iterations, Agarwal et al. scheme never converges, the CR scheme converges in 10 iterations and the SP scheme converges in 5 iterations.

3) Taking $\alpha_{n}=\beta_{n}=\gamma_{n}=\frac{1}{(1+n)^{\frac{1}{4}}}$ and $x_{\mathrm{o}}=0.8$, we obtain that the Mann scheme converges in 14 iterations, the Ishikawa scheme converges in 27 iterations, the Noor scheme converges in 9 iterations, Agarwal et al. scheme converges in 10 iterations, the CR scheme converges in 8 iterations and the SP scheme converges in 8 iterations.

\subsection{Goat Problem}

1) For $x_{0}=0.8$, the Picard scheme converges to a fixed point in 13 iterations, the Mann scheme converges in 90 iterations, the Ishikawa scheme converges in 61 iterations, the Noor scheme converges in 48 iterations, Agarwal et al. scheme converges in 11 iterations, the SP scheme converges in 11 iterations and the CR scheme 
converges in 8 iterations.

2) Taking initial guess $x_{0}=0.6$ (away from the fixed point), the Picard scheme converges to a fixed point in 14 iterations, the Mann scheme converges in 104 iterations, the Ishikawa scheme converges in 71 iterations, the Noor scheme converges in 57 iterations, Agarwal et al. scheme converges in 12 iterations, the SP scheme converges in 12 iterations and the CR scheme converges in 9 iterations.

3) Taking $\alpha_{n}=\beta_{n}=\gamma_{n}=\frac{1}{(1+n)^{\frac{1}{4}}}$ and $x_{0}=0.8$, we obtain that Mann scheme converges in 29 iterations, the Ishikawa scheme converges in 18 iterations, Noor scheme converges in 14 iterations, Agarwal et al. scheme converges in 10 iterations, the SP scheme converges in 7 iterations and the $\mathrm{CR}$ scheme converges in 7 iterations.

\section{Conclusions}

For decreasing functions, we conclude the followings:

1) Picard and Agarwal et al. schemes do not converge for $\alpha_{n}=\frac{1}{(1+n)^{\frac{1}{2}}}$, rate of convergence of the SP scheme is better than other iterative schemes, while CR and Mann schemes shows equivalence. Also, Noor scheme is faster than Ishikawa iteration.

2) On increasing the value of $m$, Mann, Ishikawa, Noor, SP and CR schemes require more number of iterations to converge.

3) For initial guess nearer to the fixed point, Mann scheme, Noor scheme shows an increase, while Ishikawa scheme shows an decrease in the number of iterations to converge. Of course, the CR scheme shows no change.

4) The speed of iterative schemes depends on $\alpha_{n}$ and $\beta_{n}$. If we increase the value of $\alpha_{n}$ and $\beta_{n}$, the fixed point is obtained in more number of iterations for all schemes. Agarwal et al. scheme converges for increased value of $\alpha_{n}$ i.e for $\alpha_{n}=\frac{1}{(1+n)^{\frac{1}{2}}}$.

In this case, increasing order of rate of convergence for iterative schemes is Ishikawa, Mann, Noor, Agarwal et al., SP and CR scheme.

For increasing functions, we conclude the followings:

1) Increasing order of rate of convergence for iterative schemes is Mann, Ishikawa, Noor, Picard, Agarwal et al., $\mathrm{SP}$ and CR scheme.

2) For initial guess away from the fixed point, the number of iterations increases in each iterative scheme. Hence, closer the initial guess to the fixed point, quicker the result is achieved.

3) If we increase the value of $\alpha_{n}$ and $\beta_{n}$, the fixed point is obtained in less number of iterations for all schemes. Except CR iterative scheme which remains unaffected.

For solution of cubic equation, we conclude the followings:

1) Picard and Agarwal et al. iterative schemes do not converge for $\alpha_{n}=\frac{1}{(1+n)^{\frac{1}{2}}}$ and rate of convergence of SP iterative scheme is better than Ishikawa, Noor, Mann and CR schemes.

2) For initial guess away from the solution, the number of iterations increases in each iterative scheme.

3 ) If we increase the value of $\alpha_{n}$ and $\beta_{n}$, the solution is obtained in less number of iterations for Noor and Ishikawa schemes while solution is obtained in more number of iterations for Mann, SP and CR schemes. Agarwal et al. iteration converges for increased value of $\alpha_{n}$ In this case, increasing order of rate of convergence for iterative schemes is Ishikawa, Mann, Agarwal et al., Noor and CR scheme while CR and SP schemes show equivalence.

For the goat problem we conclude the followings:

1) Increasing order of rate of convergence for iterative schemes is Mann, Ishikawa, Noor, Picard, SP and CR scheme while SP and Agarwal et al. schemes show equivalence.

2) For initial guess away from the fixed point, the number of iterations increases in each iterative scheme. Hence, closer the initial guess to the fixed point, quicker the result is achieved.

3) If we increase the value of $\alpha_{n}$ and $\beta_{n}$, the fixed point is obtained in less number of iterations for all schemes. In this case increasing order of rate of convergence for iterative schemes is Mann, Ishikawa, Noor, Agarwal et al. and CR scheme while CR and SP schemes show equivalence.

\section{REFERENCES}

[1] W. R. Mann, "Mean Value Methods in Iteration," Proceedings of the American Mathematical Society, Vol. 4, No. 3, 1953, pp. 506-510. doi:10.1090/S0002-9939-1953-0054846-3

[2] S. Ishikawa, "Fixed Points by a New Iteration Method," Proceedings of the American Mathematical Society, Vol. 44, 1974, pp. 147-150. doi:10.1090/S0002-9939-1974-0336469-5

[3] R. P. Agarwal, D. O'Regan and D. R. Sahu, "Iterative Construction of Fixed Points of Nearly Asymptotically Nonexpasive Mappings," Journal of Nonlinear and Convex Analysis, Vol. 8, No. 1, 2007, pp. 61-79.

[4] M. A. Noor, "New Approximation Schemes for General Variational Inequalities," Journal of Mathematical Analysis and Applications, Vol. 251, No. 1, 2000, pp. 217-229. 


\section{doi:10.1006/jmaa.2000.7042}

[5] W. Pheungrattana and S. Suantai, "On the Rate of Convergence of Mann, Ishikawa, Noor and SP Iterations for Continuous on an Arbitrary Interval," Journal of Computational and Applied Mathematics, Vol. 235, No. 9, 2011, pp. 3006-3914. doi:10.1016/j.cam.2010.12.022

[6] T. Zamfirescu, "Fixed Point Theorems in Metric Spaces," Archiv der Mathematik, Vol. 23, No. 1, 1972, pp. 292298. doi:10.1007/BF01304884

[7] V. Berinde, "On the Convergence of the Ishikawa Iteration in the Class of Quasi Contractive Operators," Acta Mathematica Universitatis Comenianae, Vol. 73, No. 1, 2004, pp. 119-126.

[8] Z. Q. Xue, "Remarks on Equivalence among Picard, Mann and Ishikawa Iterations in Normed Spaces. Fixed Point Theory and Applications, Vol. 2007, 2007, Article ID: 61434.

[9] B. E. Rhoades and S. M. Soltuz, "On the Equivalence of Mann and Ishikawa Iteration Methods," International Journal of Mathematics and Mathematical Sciences, Vol. 2003, 2003, pp. 451-459. doi:10.1155/S0161171203110198

[10] B. E. Rhoades, and S. M. Soltuz, "The Equivalence of the Mann and Ishikawa Iteration for Non-Lipschitzian Operators," International Journal of Mathematics and Mathematical Sciences, Vol. 42, 2003, pp. 2645-2652. doi:10.1155/S0161171203211418

[11] B. E. Rhoades and S. M. Soltuz, "The Equivalence between the Convergences of Ishikawa and Mann Iterations for Asymptotically Pseudo-Contractive Map," Journal of Mathematical Analysis and Applications, Vol. 283, 2003, pp. 681-688. doi:10.1016/S0022-247X(03)00338-X

[12] B. E. Rhoades and S. M. Soltuz, "The Equivalence of Mann and Ishikawa Iteration for a Lipschitzian Psi-Uniformly Pseudocontractive and Psi-Uniformly Accretive Maps, Tamkang Journal of Mathematics, Vol. 35, 2004, pp. 235-245.

[13] B. E. Rhoades and S. M. Soltuz, "The Equivalence between the Convergences of Ishikawa and Mann Iterations for Asymptotically Nonexpansive Maps in the Intermediate Sense and Strongly Successively Pseudocontractive Maps," Journal of Mathematical Analysis and Applica- tions, Vol. 289, No. 1, 2004, pp. 266-278. doi:10.1016/j.jmaa.2003.09.057

[14] B. E. Rhoades and S. M. Soltuz, "The Equivalence between Mann-Ishikawa Iterations and Multistep Iteration," Nonlinear Analysis, Vol. 58, No. 1-2, 2004, pp. 219-228. doi:10.1016/j.na.2003.11.013

[15] S. M. Soltuz, "The Equivalence of Picard, Mann and Ishikawa Iterations Dealing with Quasi-Contractive Operators," Mathematical Communications, Vol. 10, 2005, pp. 81-89.

[16] S. M. Soltuz, "The Equivalence between Krasnoselskij, Mann, Ishikawa, Noor and Multistep Iterations," Mathematical Communications, Vol. 12, 2007, pp. 53-61.

[17] R. Chugh and V. Kumar, "Strong Convergence of SP Iterative Scheme for Quasi-Contractive Operators," International Journal of Computer Applications, Vol. 31, No. 5, 2011, pp. 21-27.

[18] B. E. Rhoades, "Comments on Two Fixed Point Iteration Methods," Journal of Mathematical Analysis and Applications, Vol. 56, 1976, pp. 741-750. doi:10.1016/0022-247X(76)90038-X

[19] S. L. Singh, "A New Approach in Numerical Praxis," Progress of Mathematics (Varanasi), Vol. 32, No. 2, 1998, pp. 75-89.

[20] V. Berinde, "Iterative Approximation of Fixed Points," Springer-Verlag, Berlin, 2007.

[21] V. Berinde, "Picard Iteration Converges Faster than Mann Iteration Iteration for a Class of Quasi-Contractive Operators," Fixed Point Theory and Applications, Vol. 2, 2004, pp. 97-105. doi:10.1155/S1687182004311058

[22] Y. Qing and B. E. Rhoades, "Comments on the Rate of Convergence between Mann and Ishikawa Iterations Applied to Zamfirescu Operators," Fixed Point Theory and Applications, Vol. 2008, 2008, Article ID: 387504.

[23] L. B. Ciric, B. S. Lee and A. Rafiq, "Faster Noor Iterations," Indian Journal of Mathematics, Vol. 52, No. 3, 2010, pp. 429-436.

[24] N. Hussian, A. Rafiq, D. Bosko and L. Rade, "On Rate of Convergence of Various Iterative Schemes," Fixed Point Theory and Applications, Vol. 45, 2011, pp. 1-6. 